
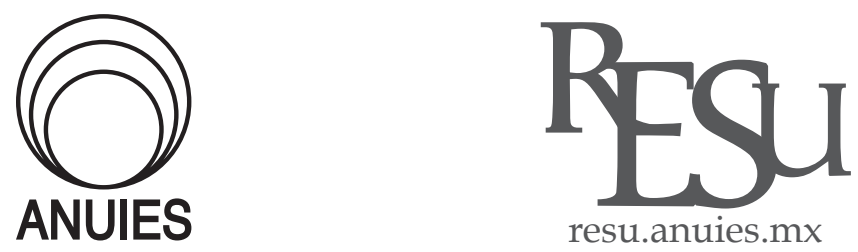

REVITA DEL
EDICACIÓN
SUPERIOR

ARTí́cUlO

\title{
Percepción de mejora de las habilidades comunicativas en estudiantes universitarios
}

\section{A Perception of Communication Skills improvement in University Students}

\section{Carmen M. Hernández-Jorge* y Carmen Marina de la Rosa Curbero**}

* Departamento de Psicología Evolutiva y de la Educación de la Universidad de La Laguna. San Cristóbal de La Laguna. España.

Recibido el 29 de marzo de 2017; aceptado el 27 de junio de 2018.

\section{Resumen}

El entrenamiento sistematizado de las habilidades comunicativas es un procedimiento eficaz para adquirirlas. Este trabajo trata de comprobar la mejora en la percepción de estas habilidades en estudiantes universitarios de carreras de apoyo, tras realizar un programa de entrenamiento. Participaron 274 estudiantes universitarios. Se utilizó un diseño Splitplot. Los resultados indican que los estudiantes entrenados mejoran en sus habilidades comunicativas frente a los no entrenados. Se concluye que este programa es un buen instrumento para mejorar las habilidades de comunicación en los estudiantes universitarios, lo cual va en la línea de diferentes estudios sobre la bondad de estos programas.

Palabras clave: Comunicación interpersonal, Habilidades comunicativas, Entrenamiento, Efectividad a corto plazo, Estudiantes universitarios. 


\begin{abstract}
The systematic training is the most efficient process to acquire appropriate communication skills. This paper aims to test the effectiveness of a training program in communication skills applied to university students. 274 students participated. The study used a Split-plot design. The results indicate that trained students perceive themselves with more communication skills. This communication skills training is a good tool to improve, in a shortterm, these skills in university students. These results are in line with several studies on the effectiveness of systematic training programs in communication skills.
\end{abstract}

Keywords: Interpersonal communication; Communication skills, Training, Short-term effectiveness; University students.

\title{
Introducción
}

L a comunicación interpersonal es un aspecto fundamental de la condición humana y una característica que nos define como tales. Inclusive, las personas que no interactúan de manera significativa con los demás suelen ser condenadas al ostracismo, lo que tiende a conducirles a la soledad, la infelicidad y la depresión (Williams y Zadro, 2001). Además de esto, sabemos que las personas que poseen habilidades interpersonales están más satisfechas con sus relaciones cercanas (Miczo, Segrin y Allspach, 2001), afrontan más fácilmente el estrés, se adaptan y ajustan mejor a la vida y son menos propensas a sufrir depresión, soledad o ansiedad (Segrin y Flora, 2000). En general, parece que las personas socialmente competentes son más resistentes a los efectos negativos de las crisis que suceden en sus vidas (Segrin, 2000), por lo que podría decirse que poseer habilidades interpersonales y de comunicación es un recurso útil ante la adversidad).

También en el ámbito laboral son relevantes estas habilidades, no en vano, la mayoría de profesionales trabajan en colaboración, tanto presencialmente como mediante espacios colaborativos virtuales (Roberts y Ruts, 1994; Prendes y Solano, 2008); sobre todo, lo hacen los que utilizan la comunicación como medio fundamental para alcanzar sus objetivos, tales como los profesionales de la salud, los profesionales de apoyo o los docentes (Camacho y Sáenz, 2000; Dickson, Hargie y Morrow, 1997; autora, 1999; Turner y Merriman, 2005). Además, utilizar habilidades comunicativas adecuadas se relaciona positivamente con la competencia profesional, de manera que los 
usuarios perciben más competentes a los profesionales que las poseen (Colliver, Swartz, Robbs y Cohen, 1999; Greene y Burleson, 2003). Finalmente conocemos que el buen uso de las habilidades comunicativas contribuye a la mejora de la salud, el comportamiento o el rendimiento de los usuarios (Camacho y Sáenz, 2000; Good y Brophy, 1996; Hargie, 2011).

Estas habilidades suelen adquirirse de forma natural en contacto con entornos enriquecidos que permitan ejercitarlas; pero otra manera de adquirirlas es mediante el entrenamiento sistematizado de las mismas, el cual se dirige explícita e intencionalmente a proporcionar habilidades de comunicación interpersonal a profesionales tanto en formación como en ejercicio. Bajo esta característica se engloban diferentes métodos de entrenamiento, que se diferencian en los objetivos que persiguen y en los procedimientos que utilizan, lo cuales van desde las charlas informativas hasta la microenseñanza (autora, 2005).

Existen cuatro grupos de métodos generales de entrenamiento en las habilidades de comunicación. Un primer grupo se centra en la acción y se basa en la microenseñanza, que consiste en descomponer el contenido en pequeñas unidades fáciles de entender y de practicar, suele utilizar situaciones simuladas que proporcionan la sensación de seguridad en los participantes, ya que son controlables por ellos (Gimeno, 2008); se lleva a cabo con grupos reducidos, creando un contexto facilitador del aprendizaje (Lozano, 2005); sus objetivos son de acción y se ofrece retroalimentación a los participantes de su actuación (Paulley y Benwari, 2014).

Otro grupo de métodos se centra en la cognición y su objetivo es que los participantes reflexionen acerca de por qué y para qué se aplican las habilidades comunicativas a las diversas situaciones interpersonales que experimentan, y se basan en la teoría de la información y de la teoría constructivista del aprendizaje (Zade, Akbari y Khanzade, 2014); desde estas perspectivas teóricas, el entrenamiento trata de integrar el conocimiento de la comunicación (qué), la adquisición de las habilidades (cómo) y la contextualización de las mismas (a quién y dónde se comunica), así como generar un proceso activo de aprendizaje, de manera que los participantes contextualicen las habilidades adquiridas a diversas situaciones ya sus características personales.

Un tercer grupo de métodos se centra en el componente emocional de la comunicación interpersonal y persigue que los participantes conozcan mejor sus propias motivaciones, emociones o sentimientos y su influencia en los otros; este tipo de métodos consigue que los participantes se impliquen y comprometan en su proceso de aprendizaje, compartiendo sus percepciones, actitudes y motivaciones (Aksoy y Baran, 2010). 
Finalmente, un cuarto grupo de métodos se basa en la aplicación de la Tecnología de la Información y la Comunicación (TIC) e incluye simulaciones de ordenador, sistemas expertos, sistema de video interactivo y teleformación (Ehrlich y Miller, 2009; Minz, Branch, March y Lerman, 2012). En general, las aproximaciones actuales integran estos métodos, de manera que los participantes se forman en destrezas, reflexionan sobre la comunicación, trabajan los aspectos emocionales y utilizan la tecnología de la información y la comunicación (Peñacoba, Ardoy, González-Gutiérrez, Moreno-Rodríguez y Martínez, 2003; Hussainy,Stykes y Duncan, 2012; Choi, Song y Oh, 2015).

Actualmente el entrenamiento sistematizado en habilidades comunicativas se aplica en ámbitos y a colectivos diversos (relaciones laborales y organizacionales, educación; salud mental; etc.) y se ha evaluado desde una triple perspectiva: la de los participantes, la de observadores externos cualificados y la de los usuarios que interactúan con los profesionales entrenados, contemplándose diferentes criterios para evaluar su eficacia. Los estudios señalan que los participantes en el entrenamiento suelen percibir beneficios en él y la posibilidad de transferencia a situaciones reales (Baker, Gustafson, Beaubien, Salas y Barach, 2005). También los profesionales entrenados en habilidades de comunicación perciben mejoras en las mismas después del entrenamiento y mayor competencia en ellas que aquellos no entrenados (Meunier et al., 2013; Pereira-Guizzo, Del Prette y Del Prette, 2012). Sin embargo, los datos son menos concluyentes cuando se observa la efectividad a largo plazo y la transferencia a otras situaciones. Los resultados señalan que, si bien se dan mejoras en las habilidades inmediatamente después del entrenamiento, también parece que su nivel de ejecución decrece con el tiempo (Moral y Ovejero, 2005). Respecto a la generalización de los resultados algunas investigaciones señalan que la transferencia a situaciones naturales se produce, sobre todo, cuando se aumenta paulatinamente el tiempo entre las sesiones de entrenamiento (Francisco y Hanley, 2012), o cuando se aplican apoyos sistemáticos para identificar las habilidades entrenadas en las situaciones cotidianas (Tauber, Wallace y Lecomte, 2000). Tampoco los resultados son concluyentes cuando se compara este tipo de entrenamiento con otros, ya que algunos estudios manifiestan que el entrenamiento sistematizado genera beneficios frente a otros métodos, mientras que otros señalan que no son tales (Kroeger, Schultz y Newson, 2007; Rees, Sheard y McPherson, 2004). La falta de resultados concluyentes estriba en que la mayoría de estudios no informan suficientemente de las habilidades comunicativas entrenadas y en que, en ocasiones, no hay correspondencia entre éstas y los instrumentos utilizados para evaluarlas (Cegala y Lenzmeier, 2002). 
También se ha estudiado la influencia de distintas variables del propio método de entrenamiento en la adquisición, la generalización y el mantenimiento de sus ganancias. Por ejemplo, no parece haber diferencia en el uso de pacientes simulados (actores externos) y el juego de roles (realizado por los participantes en el taller) para la efectividad del entrenamiento (Lane y Rollnick, 2007), al tiempo que parece incrementarse la eficacia del mismo cuando se integran diferentes técnicas (distintos escenarios sociales, mediación de pares, habilidades cognitivas...) (Aksoy y Baran, 2010; Flynn y Healy, 2012; Gil, Cantero y Antino 2013; Wang, Cui, y Parrila, 2011) o cuando el entrenamiento forma parte de otro programa más general de tipo curricular o comunitario (Chan, 2006; Kopelowicz, Liberman y Zarate, 2006). En nuestro caso, el programa de entrenamiento forma parte de una asignatura más amplia, en la que se imparte toda la fundamentación teórica sobre habilidades interpersonales y de comunicación.

Además de los aspectos relacionados con el proceso de entrenamiento, consideramos que sería interesante ahondar en distintas características de los participantes, ya que podrían modular las ganancias que experimentan con él. Nos referimos a distintas variables intrapersonales que influyen en el proceso de formación y aprendizaje, tales como las creencias de autoeficacia formulada en la teoría social cognitiva de Bandura $(1987,1997)$. De hecho, se dan mejoras en la autoeficacia social en estudiantes universitarios que participan en programas de habilidades sociales. Una investigación evidencia que los estudiantes que reciben entrenamiento en habilidades sociales mejoran sus creencias de autoeficacia, independientemente de la modalidad de entrenamiento utilizada frente a los estudiantes no entrenados (Olaz y Pérez, 2012). Otro estudio respecto a la autoeficacia social académica en estudiantes universitarios de ciencias de la salud, encuentra una relación positiva entre los sentimientos de autoeficacia social con el disfrute vital y la esperanza, y de forma negativa con la ansiedad y la vergüenza a la hora de buscar ayuda académica o participar en clase (Sánchez, 2013). Esto puede evidenciar la importancia de considerar la autoeficacia social y académica como moduladores de la percepción de la ganancia en programas de entrenamiento en habilidades de comunicación, teniendo en cuenta que la competencia de comunicación interpersonal tiene relaciones con la percepción de autoeficacia.

Al margen de la perspectiva utilizada, parece que la efectividad del entrenamiento sistematizado en habilidades de comunicación es generalmente satisfactoria (López, 2013), es la intervención más empleada para ayudar a las personas a desarrollar relaciones significativas y a promover su integración social en la comunidad (Gil et al., 2013) y es una estrategia ampliamente utili- 
zada con estudiantes universitarios (Bylund, Brown, Lubrano di Ciccone, Levin, Gueguen, Hill y Kissane, 2008; Tiuraniemi, La“a“ra“, Kyro, y Lindeman, 2011; Hussainy, Stykes y Duncan, 2012; Mort y Hansen, 2010).

El programa de entrenamiento que aquí se presenta tiene como objetivo general enseñar y mejorar las habilidades de comunicación interpersonal de estudiantes universitarios en situaciones profesionales. Los objetivos específicos integran el conocimiento, la acción y la emoción, tal como orientan algunos investigadores (Flynn y Healy, 2012; Wang et al., 2011; Zade et al., 2014). Un ejemplo de objetivos de conocimiento es "Favorecer la reflexión y el análisis del acto comunicativo, su complejidad y su carácter multidimensional"; un ejemplo de objetivos de acción es "Diseñar y aplicar las habilidades de comunicación en diferentes situaciones profesionales: asesoramiento individualizado y acciones formativas" y finalmente un ejemplo de objetivo emocional es "Propiciar actitudes comunicativas adecuadas".

El programa consta de las siguientes fases generales:

- Fase de información teórica: en la que se fundamenta la comunicación interpersonal, sus variables, elementos y procesos.

- Fase de información técnica: donde se ofrece una taxonomía concreta de habilidades referidas al asesoramiento individual y la docencia.

- Fase de ejemplificación o modelado: en ella se utilizan ejemplos concretos donde se observa la aplicación de diferentes habilidades.

- Fase de elaboración: donde los participantes elaboran situaciones similares a las ejemplificadas anteriormente.

- Fase de simulación: en ella los participantes realizan un juego de roles con la situación elaborada previamente, que es grabada en vídeo.

- Fase de visionado: donde los participantes observan el vídeo grabado y se realiza en grupo.

- Fase de evaluación: en la que los participantes valoran cada una de las sesiones, el entrenamiento completo, a la monitora y su propia implicación y aprendizaje.

Además de la información teórica, que constaba de nueve horas, el programa se desarrolló en nueve sesiones presenciales (dos horas cada una), que corresponden a cinco unidades, referidas a cinco contenidos temáticos sobre la comunicación interpersonal. La temporalización de las sesiones se distribuyó de la siguiente manera:

- Unidad 1: Conociendo las habilidades comunicativas (2 horas presenciales).

- Unidad 2: Comenzando a trabajar la comunicación interpersonal (4 horas presenciales).

- Unidad 3: Escuchar para comprender (4 horas presenciales). 
- Unidad 4: Comunicándonos con un grupo (6 horas presenciales).

- Unidad 5: Valorando el entrenamiento (2 horas).

Los procedimientos instruccionales que se utilizaron para realizar el entrenamiento fueron diversos e iban desde los debates, reflexiones sobre modelos observables o simbólicos hasta el juego de roles o de metáforas vivenciales.

El presente estudio tiene como objetivo comprobar la percepción de mejora en las habilidades comunicativas que tienen los estudiantes universitarios de carreras de apoyo, tras la participación en un programa de entrenamiento en habilidades de comunicación. Lo cual situaría este estudio en el criterio de eficacia a corto plazo (Meunier et al., 2013; Pereira-Guizzo, Del Prette y Del Prette, 2012).

\section{Método}

\section{Participantes}

Los participantes en este estudio fueron 274 estudiantes universitarios, de los cuales 169 son mujeres y 105 hombres, con un rango de edad de 18 a 35 años. Los estudiantes cursaban estudios de grado y posgrado y se distribuyen tal como se recoge en la tabla 1 .

Tabla 1

Datos de los participantes en el estudio

\begin{tabular}{lcccc}
\hline Grupos & Número & Hombres & Mujeres & $\begin{array}{c}\text { Media de } \\
\text { edad }\end{array}$ \\
\hline Intervención & 85 & 14 & 71 & 24.7 \\
\hline Control de Apoyo & 93 & 18 & 75 & 22.1 \\
\hline Control de no Apoyo & 96 & 73 & 23 & 22.4 \\
\hline Total & $\mathbf{2 7 4}$ & $\mathbf{1 0 5}$ & $\mathbf{1 6 9}$ & $\mathbf{2 3 . 1}$ \\
\hline
\end{tabular}

\section{Instrumentos}

Se utilizó el Cuestionario de Habilidades Comunicativas (HABICOM), que recoge la percepción sobre el uso de las habilidades comunicativas. El cuestionario consta de 54 preguntas. Las 10 primeras recogen información sociodemográfica (género, edad, estado civil, nacionalidad, estudios, titulación académica, trabajo actual o no, ocupaciones anteriores y formación previa en 
habilidades de comunicación) y las 34 restantes versan sobre las habilidades y actitudes comunicativas, un ejemplo de pregunta es la siguiente: “Mantengo la expresión gestual (Utilizo gestos con las manos o con el cuerpo que acompañan el discurso y que suelen estar acordes con lo que digo)." El formato de respuesta es una escala tipo Likert con cinco niveles, donde 1 es no lo utilizo nunca y 5 lo uso siempre.

Este cuestionario ha sido elaborado por las autoras del trabajo. Ha obtenido una alta consistencia interna $(\alpha=.91)$ y fue sometido a una prueba test-retest (con 3 meses de diferencia), estableciéndose que los estudiantes no muestran diferencias significativas $(p<.05)$ entre ambas mediciones. El análisis factorial realizado arrojó los siguientes factores: 1 Generar motivación; 2 Comunicación no verbal; 3 Empatía; 4 Expresión Emocional; 5 Expresión Oral; 6 Transmisión de Información; 7 Comunicación Abierta y Auténtica y 8 Escucha.

\section{Procedimiento}

Se ha utilizado un diseño cuasi-experimental mixto, en el que se compara el grupo de intervención, al que se aplicó el programa de entrenamiento en habilidades de comunicación, con dos grupos de control. Uno de ellos afín al grupo de intervención, el cual está conformado también por estudiantes de carreras de apoyo; y otro no afín a él, formado por estudiantes de carreras diferentes a las carreras de apoyo.

La asignación a los grupos no fue aleatoria. El grupo de intervención estaba constituido por estudiantes que cursaban la asignatura de "Entrenamiento en habilidades de comunicación", en este caso estudiantes de logopedia y dos de los máster oficiales de psicología. El grupo de control, afín al de intervención, lo constituía estudiantes de titulaciones que comparten un centro de estudios con el grupo de intervención y en las que no se imparte esta asignatura, concretamente la carrera de psicología y otros dos máster oficiales de la facultad de psicología de la Universidad de La Laguna. El grupo de control no afín al de intervención lo conformaban estudiantes de distintas carreras no consideradas de apoyo y que accedieron desinteresadamente a participar en la investigación.

El programa de intervención fue la variable independiente y las variables criterio fueron las habilidades de comunicación recogidas en el cuestionario descrito. Éste se aplicó antes de la intervención en los tres grupos por la misma persona. Se pedía a los participantes su identificación mediante un alias y se informó sobre la confidencialidad de los datos. Una vez aplicado el 
cuestionario, se realizó el entrenamiento al grupo de intervención, incluido en las asignaturas de su plan de estudios y realizado por la misma monitora. La segunda medición se obtuvo inmediatamente después de la intervención en los tres grupos participantes en el estudio.

\section{Análisis Estadístico}

Se utilizó el análisis de varianza de medidas repetidas (Split-plot) y la prueba $t$ para muestras relacionadas del paquete estadístico SPSS/ PC + (versión 17.0). Además, se calculó el tamaño del efecto ( $d$ de Cohen).

\section{Resultados}

En primer lugar se mostrarán los resultados de las diferencias intragrupo de cada uno de ellos en las dos mediciones realizadas y posteriormente las diferencias entre los grupos de intervención y los de control.

\section{Respecto a las diferencias intragrupo}

En el grupo de intervención los resultados revelaron diferencias estadísticamente significativas $(p<.05)$ en la mayoría de los factores del cuestionario. Estos fueron, generar motivación $(\mathrm{M}=-0.25, \mathrm{DT}=0.91) ; \mathrm{t}(84)=-2.58, \mathrm{p}=.01$; comunicación no verbal $(\mathrm{M}=-0.20$, $\mathrm{DT}=0.58) ; \mathrm{t}(84)=-3.12, \mathrm{p}=.00$; empatía $(\mathrm{M}=-0.20, \mathrm{DT}=0.54) ; \mathrm{t}(84)=-3.44, \mathrm{p}=.00$; expresión oral $(\mathrm{M}=-0.27, \mathrm{DT}=0.66)$; $\mathrm{t}(84)=-3.71, \mathrm{p}=.00$; transmisión de información $(\mathrm{M}=-0.19, \mathrm{SD}=0.68) ; \mathrm{t}(84)=$ -2.62, $\mathrm{p}=.01$.; comunicación abierta y auténtica $(\mathrm{M}=-0.22, \mathrm{SD}=0.53)$; $\mathrm{t}(84)=$ $-3.89, \mathrm{p}=.00$. y recabar información y escuchar $(\mathrm{M}=-0.25, \mathrm{SD}=0.67) ; \mathrm{t}(84)=$ $-3.39, \mathrm{p}=.00$.

Parece que los participantes del grupo de intervención mejoran significativamente en la percepción que tienen de sus habilidades de comunicación después del entrenamiento, considerándose más capaces de partir de las necesidades del otro, generar expectativas positivas, ponerse su lugar, usar el lenguaje verbal y no verbal adecuadamente, transmitir la información de forma clara, ser congruentes, querer participar de la comunicación y escuchar. Sin embargo, el tamaño del efecto es pequeño o moderado (véase tabla 2). 
Tabla 2

Resultados del Grupo de Intervención antes y después de la misma

\begin{tabular}{|c|c|c|c|c|c|c|c|}
\hline Factores & $\begin{array}{c}\text { M. } \\
\text { Pretest. }\end{array}$ & $\begin{array}{c}\text { M. } \\
\text { Postest }\end{array}$ & Media & D. T. & $t$ & $\begin{array}{c}\text { Sig. } \\
\text { (bilateral) }\end{array}$ & d \\
\hline 1. Generar motivación & 4.00 & 4.25 & -0.25 & 0.91 & -2.58 & .01 & .27 \\
\hline $\begin{array}{l}\text { 2. Comunicación no } \\
\text { verbal }\end{array}$ & 4.09 & 4.29 & -0.20 & 0.58 & -3.12 & .00 & .32 \\
\hline 3. Empatía & 4.24 & 4.44 & -0.20 & 0.54 & -3.44 & .00 & .35 \\
\hline 4. Expresiónemocional & 3.89 & 4.01 & -0.11 & 0.69 & -1.52 & .13 & .16 \\
\hline 5. Expresión oral & 3.81 & 4.08 & -0.27 & 0.66 & -3.71 & .00 & .38 \\
\hline $\begin{array}{l}\text { 6. Transmisión de } \\
\text { información }\end{array}$ & 3.79 & 3.98 & -0.19 & 0.68 & -2.62 & .01 & .27 \\
\hline $\begin{array}{l}\text { 7. Comunicación abierta y } \\
\text { auténtica }\end{array}$ & 3.93 & 4.15 & -0.22 & 0.53 & -3.89 & .00 & .39 \\
\hline 8. Escucha & 3.96 & 4.21 & -0.25 & 0.67 & -3.39 & .00 & .35 \\
\hline
\end{tabular}

Respecto al grupo control afín al de intervención (estudiantes de carreras de apoyo), los resultados revelaron que no existen diferencias estadísticamente significativas entre las dos mediciones realizadas, salvo en dos factores: la expresión emocional $(\mathrm{M}=0.21, \mathrm{SD}=0.79) ; \mathrm{t}(92)=2.53, \mathrm{p}=.01$ y la escucha $(\mathrm{M}=$ $0.18, \mathrm{SD}=0.59$ ); $\mathrm{t}(92)=2.95, \mathrm{p}=.00$ (véase tabla 3 ). Ambas diferencias se traducen en un decremento en la puntuación obtenida en la segunda medición. El tamaño del efecto en este grupo es pequeño en todos los factores.

Tabla 3

Resultados del Grupo de Control afín al de intervención en las dos mediciones realizadas

\begin{tabular}{|c|c|c|c|c|c|c|c|}
\hline Factores & $\begin{array}{c}\text { M. } \\
\text { Pretest. }\end{array}$ & $\begin{array}{c}\text { M. } \\
\text { Postest }\end{array}$ & Media & D. T. & $\mathbf{t}$ & $\begin{array}{c}\text { Sig. } \\
\text { (bilateral) }\end{array}$ & d \\
\hline 1. Generar motivación & 3.93 & 3.91 & 0.01 & 0.54 & 0.26 & .80 & .03 \\
\hline $\begin{array}{l}\text { 2. Comunicación no } \\
\text { verbal }\end{array}$ & 4.02 & 3.98 & 0.04 & 0.63 & 0.56 & .58 & .06 \\
\hline 3. Empatía & 4.41 & 4.32 & 0.09 & 0.53 & 1.72 & .09 & .18 \\
\hline 4. Expresión emocional & 3.82 & 3.62 & 0.21 & 0.79 & 2.53 & .01 & .25 \\
\hline 5. Expresión oral & 3.67 & 3.95 & -0.27 & 1.52 & -1.74 & .09 & .18 \\
\hline $\begin{array}{l}\text { 6. Transmisión de } \\
\text { información }\end{array}$ & 3.59 & 3.71 & -0.12 & 0.62 & -1.88 & .06 & .19 \\
\hline $\begin{array}{l}\text { 7. Comunicación abierta y } \\
\text { auténtica }\end{array}$ & 3.78 & 3.86 & -0.08 & 0.66 & -1.13 & .26 & .12 \\
\hline 8. Escucha & 4.06 & 3.88 & 0.18 & 0.59 & 2.95 & .00 & .29 \\
\hline
\end{tabular}

En el grupo control no afín al de intervención (estudiantes de carreras que no son de apoyo), no se produjeron cambios significativos al comparar los dos 
momentos de la medición (véase tabla 4). Los participantes no cambiaron la percepción que tenían de sus habilidades y actitudes comunicativas. También en esta ocasión el tamaño del efecto es pequeño en todos los factores.

Tabla 4

Resultados del Grupo de Control afín al de intervención en las dos mediciones realizadas

\begin{tabular}{lccccccc}
\hline Factores & $\begin{array}{c}\text { M. } \\
\text { Pretest. }\end{array}$ & $\begin{array}{c}\text { M. } \\
\text { Postest }\end{array}$ & Media & D. T. & t & $\begin{array}{c}\text { Sig. } \\
\text { (bilateral) }\end{array}$ & d \\
\hline 1. Generar motivación & 3.60 & 3.66 & -0.06 & 0.87 & -0.68 & .50 & .07 \\
\hline $\begin{array}{l}\text { 2. Comunicación no } \\
\text { verbal }\end{array}$ & 3.60 & 3.55 & 0.05 & 0.60 & 0.82 & .42 & .08 \\
\hline 3. Empatía & 3.90 & 3.84 & 0.06 & 0.51 & 1.15 & .25 & .12 \\
\hline $\begin{array}{l}\text { 4. Expresión emocional } \\
\text { 5. Expresión oral }\end{array}$ & 3.27 & 3.18 & 0.09 & 0.65 & 1.41 & .16 & .14 \\
\hline $\begin{array}{l}\text { 6. Transmisión de } \\
\text { información }\end{array}$ & 3.45 & 3.45 & 0.01 & 0.59 & 0.09 & .93 & .01 \\
\hline $\begin{array}{l}\text { 7.Comunicación abierta y } \\
\text { auténtica }\end{array}$ & 3.51 & 3.46 & -0.07 & 0.49 & -1.31 & .20 & .13 \\
\hline $\begin{array}{l}\text { 8. Escucha } \\
\begin{array}{l}\text { gl=95. } \\
\text { M. pretest: Media pretest; M. postest: Media postest; D.T.: Desviación Típica; gl=grados de libertad }\end{array}\end{array}$
\end{tabular}

\section{Respecto a las diferencias intergrupo}

En relación a las habilidades de comunicación, hemos de plantear que el grupo de intervención es semejante al grupo de control afín, sin embargo no lo es respecto al grupo de control no afín. Al mismo tiempo, también es necesario señalar que los dos grupos de control son diferentes entre sí antes de la intervención en las variables señaladas.

A la hora de comprobar las diferencias antes y después del grupo de intervención en relación a los grupos control realizamos contrastes univariados, los cuales mostraron diferencias estadísticamente significativas $(p<.05)$ a favor del grupo de intervención en los siguientes factores: generar motivación, expresión oral, transmisión de información y en la comunicación abierta y auténtica.

Las ganancias en el grupo de intervención frente a los grupos de control estriban en que sus participantes perciben que manejan, más que antes de la intervención, habilidades para motivar al interlocutor (GI M Pretest $=4, \mathrm{M}$ Postest 4.25; CA: M Pretest= 3.93 M Postest 3.91; CNA: M Pretest=3.60, M Postest 3.66), se perciben mejores en su expresión oral (GI M Pretest=3.81, M Postest 4.08; CA: M Pretest=3.67 M Postest 3.95; CNA: M Pretest=3.45, M Postest 3.45), incrementan la claridad en la transmisión de la información (GI 
M Pretest= 3.79, M Postest 3.98; CA: M Pretest= 3.59 M Postest 3.71; CNA: M Pretest=3.40, M Postest 3.46) y perciben que se comunican de forma más abierta y auténtica (GI M Pretest=3.93, M Postest 4.15; CA: M Pretest=3.78 M Postest 3.86; CNA: M Pretest=3.51, M Postest 3.49) (véase tabla 5).

Tabla 5

Diferencias entre el grupo de intervención y los dos grupos de control antes y después de la intervención

\begin{tabular}{|c|c|c|c|c|c|c|c|c|c|c|c|c|}
\hline \multirow{3}{*}{ Factores } & \multicolumn{6}{|c|}{ Pretest } & \multicolumn{6}{|c|}{ Postest } \\
\hline & \multicolumn{2}{|c|}{ GI } & \multicolumn{2}{|c|}{ CCA } & \multicolumn{2}{|c|}{ CCNA } & \multicolumn{2}{|c|}{ GI } & \multicolumn{2}{|c|}{ CA } & \multicolumn{2}{|c|}{ CNA } \\
\hline & $\mathbf{M}$ & DT & $\mathbf{M}$ & DT & $\mathbf{M}$ & DT & $\mathbf{M}$ & DT & $\mathbf{M}$ & DT & $\mathbf{M}$ & DT \\
\hline $\begin{array}{l}\text { 1. Generar } \\
\text { motivación }\end{array}$ & 4.00 & .49 & 3.93 & .53 & 3.60 & .64 & 4.25 & .88 & 3.91 & .52 & 3.66 & .91 \\
\hline $\begin{array}{l}\text { 2. Comunicación } \\
\text { no verbal }\end{array}$ & 4.09 & .60 & 4.02 & .58 & 3.60 & .64 & 4.29 & .55 & 3.98 & .65 & 3.55 & .66 \\
\hline 3. Empatía & 4.24 & .47 & 4.41 & .50 & 3.90 & .61 & 4.44 & .52 & 4.32 & .55 & 3.84 & .63 \\
\hline $\begin{array}{l}\text { 4. Expresión } \\
\text { emocional }\end{array}$ & 3.89 & .68 & 3.82 & .79 & 3.27 & .82 & 4.01 & .73 & 3.62 & .86 & 3.18 & .79 \\
\hline 5. Expresión oral & 3.81 & .61 & 3.67 & .66 & 3.45 & .68 & 4.08 & .68 & 3.95 & 1.49 & 3.45 & .66 \\
\hline $\begin{array}{l}\text { 6.Transmisión de } \\
\text { información }\end{array}$ & 3.79 & .67 & 3.59 & .62 & 3.40 & .64 & 3.98 & .62 & 3.71 & .64 & 3.46 & .66 \\
\hline $\begin{array}{l}\text { 7. Comunicación } \\
\text { abierta y } \\
\text { auténtica }\end{array}$ & 3.93 & .56 & 3.78 & .59 & 3.51 & .59 & 4.15 & .51 & 3.86 & .59 & 3.49 & .59 \\
\hline 8. Escucha & 3.96 & .62 & 4.06 & .60 & 3.70 & .69 & 4.21 & .66 & 3.88 & .65 & 3.65 & .65 \\
\hline
\end{tabular}

GI: Grupo de Intervención; CCA: Grupo de control Afín; CCNA: Grupo de Control No Afín; M Media; D.T.: Desviación Típica

\section{Discusión y Conclusiones}

El objetivo del estudio era comprobar la mejora en la percepción de las habilidades comunicativas de estudiantes universitarios de carreras de apoyo, tras la participación en un programa de entrenamiento en habilidades de comunicación. Los resultados confirman que los participantes se perciben con más habilidades para generar motivación en el interlocutor, para expresarse verbalmente (vocalizar, adecuar el volumen a la situación, etc.), para transmitir la información de manera clara y estructurada y con una mayor predisposición a comunicarse de forma abierta y auténtica. Por tanto, consideramos que este programa de entrenamiento en habilidades comunicativas es un buen instrumento para mejorar, a corto plazo, estas habilidades en los futuros profesionales de apoyo o asesoramiento, además de hacerlo con un costo y tiempo reducidos. Estos resultados van en la línea de diferentes estudios sobre las eficacia del entrenamiento sistematizado en habilidades 
comunicativas dirigidos a distintas poblaciones y ámbitos (Pereira-Guizzo et al., 2012; Meunier et al., 2013;Choi, Song y Oh 2015) y, sobre todo, con estudiantes universitarios de carreras de apoyo (Bylund, Brown, Lubrano di Ciccone, Levin, Gueguen, Hill y Kissane, 2008; Tiuraniemi, La“a“ra“, Kyro, y Lindeman, 2011; Hussainy, Stykes y Duncan, 2012; Mort y Hansen, 2010).

Por otra parte, se han producido algunos cambios hacia un empeoramiento en algunas de las habilidades de comunicación en la segunda medición en el grupo de control afín. Estos cambios van en la línea de utilizar menos la expresión de las propias emociones y la escucha. Consideramos que los estudiantes de profesiones de apoyo pueden ser más exigentes en su valoración de las habilidades que poseen, en concreto los participantes en el estudio cursaban la carrera de Psicología y de Máster de la misma rama, en la que se trabajan contenidos sobre la importancia de estas habilidades en los profesionales de la psicología. Hipotetizamos que esta pueda ser una posible explicación a este resultado.

Al mismo tiempo queremos comentar algunas limitaciones que consideramos tiene este trabajo. Una primera hace referencia a la medición. La mayoría de estudios utiliza como instrumento de medida los cuestionarios autoinformados, tal como es nuestro caso y aunque, se señalan algunas bondades psicométricas del instrumento, sabemos de las limitaciones de este tipo de apreciaciones, tal como la subjetividad. Por ello podría utilizarse en investigaciones posteriores una más objetiva, como el uso de protocolos de observación en situaciones naturales o heteroevaluaciones que realicen personas expertas.

Otra limitación es que se utiliza un criterio de eficacia a corto plazo y no toma una medida de seguimiento a medio plazo de las ganancias del entrenamiento, aspecto que no nos informa sobre si estos cambios se mantienen en el tiempo, lo cual podría ser importante como otra medida de eficacia de este tipo de entrenamiento.

Una tercera limitación es que se trabaja exclusivamente con habilidades de comunicación, obviando otras variables que podrían ser interesantes, tal como indagar en el efecto que puede tener un el programa en otras variables asociadas al adecuado desempeño profesional como la confianza y la seguridad profesional, la autocompetencia, la inteligencia emocional o la percepción de autoeficacia personal, así lo apuntan algunos trabajos revisados. En concreto, es especialmente importante resaltar el sentimiento de autoeficacia social, ya que afecta al comportamiento y ofrece un rol mediador para la adquisición y afianzamiento de las conductas, señalan entre otros Olaz y Pérez (2012).

Una cuarta limitación del estudio es que el tamaño del efecto no es amplio, quizá sería interesante revisar cada uno de los aspectos del propio programa 
de entrenamiento para ir ajustándolo en vista de incrementar la efectividad del mismo y el tamaño del efecto obtenido. Finalmente sería interesante aplicar este entrenamiento a profesionales en ejercicio para así poder comprobar si se producen estos mismos resultados o son diferentes de los obtenidos con los estudiantes.

Los resultados obtenidos en conjunto permiten enfatizar la importancia de entrenar sistemática y explícitamente las habilidades de comunicación interpersonal a los futuros profesionales de apoyo; ya que les permite reflexionar sobre la comunicación interpersonal y su importancia, incrementar la sensibilidad ante el uso de las habilidades para comunicarse y la necesidad de ponerlas en práctica. Consideramos que el presente trabajo aporta un instrumento de intervención que, aunque con limitaciones, ha sido validado empíricamente, instrumento que puede extenderse a universitarios de distintas carreras, ya que se dirige a una de las competencias transversales consideradas para el empleo y el desempeño profesional.

\section{Referencias}

Aksoy, P. \&Baran, G. (2010). Review of studies aimed at bringing social skills for children in preschool period. Procedia-Social and Behavioral Sciences, 9, 663-669.

Baker, D. P., Gustafson, S., Beaubien, J. M., Salas, E. \&Barach, P. (2005). Medical team training programs in health care. Advances in Patient Safety: fromresearch to implementation, 4, 253-267.

Bandura, A. (1987). Pensamiento y acción. Madrid: Martínez Roca.

Bandura, A. (1997). Self-efficacy: theexercise of control. New York: Freeman.

Bylund, C. , Brown, R. F., Lubrano di Ciccone, B.; Levin, T. T.; Gueguen, J. A.; Hill, C. y Kissane, D. W. (2008). Training faculty to facilitate communication skills training: Development and evaluation of a workshop. Patient Education and Counseling, 70, 430-436.

Camacho, S. \& Sáenz, O. (2000) Técnicas de comunicación eficaz para profesores y formadores. Alcoy: Marfil.

Cegala, D. J. \&Lenzmeier, S. (2002). Physician communication skills training: a review of theoretical backgrounds, objectives and skills. Medical education, 36 (11), 1004-1016.

Chan, K. (2006). Social Skills Intervention for Students with Emotional/ Behavioral Disorders: A Literature Review from the American Perspective. Educational Research and Reviews, 1, 143-149.

Choi, Y., Song, E. \& Oh, E. (2015). Effects of teaching communication skills using a video clip on a smart phone on communication competence and emotional intelligence in nursing students. Archives of Psychiatric Nursing, 29, 90-95.

Colliver, J. A.; Swartz, M. H.; Robbs, R. \& Cohen, D. (1999) Relationship between clinical competence and interpersonal and communication skills in standardized-patient assessment. Academic Medicine, 74, (3), 271-274. 
Dickson, D., Hargie, O. \& Morrow, N. (1997). Communication skills training for health professionals.Londres: Chapman \& Hall.

Ehrlich, J. \& Miller, J.R. (2009). A virtual environment for teaching social skills: AVISSS. IEEE.Computer Graphics and Applications, 29, 10-16.

Francisco, M. T. \& Hanley, G. P. (2012). An evaluation of progressively increasing intertrial intervals on the acquisition and generalization of three social skills. Journal of applied behavior analysis, 45 (1), 137-142.

Flynn, L. \& Healy, O. (2012). A review of treatments for deficits in social skills and self-help skills in autism spectrum disorder. Research in Autism Spectrum Disorders, 6, 431-441.

Gil, F., Cantero, F. J., \& Antino, M. (2013).Tendencias actuales en el ámbito de las habilidades sociales. Apuntes de Psicología, 31 (1), 51-57.

Gimeno, J. (2008). La enseñanza: su teoría y práctica (6 ${ }^{\mathrm{a}}$. Ed.). Madrid: Ediciones Akal.

Good, T. \& Brophy, J.(1996). Psicología Educativa Contemporánea. Madrid: McGraw-Hill.

Greene, J. O., \& Burleson, B. R. (2003). Handbook of communication and social interaction skills. Mahwah, NJ: Erlbaum.

Hargie, O. (2011). Skilled Interpersonal Communication: Research, Theory and Practice (5 . Ed.). London: Routledge.

Autora. (1999) Valoración de un modelo de entrenamiento en habilidades comunicativas según los participantes. Evaluación e intervención psicoeducativa: Revista interuniversitaria de psicología de la educación, 4 (1), 151-168.

Autora. (2005). Habilidades comunicativas para profesionales. Santa Cruz de Tenerife: Arte comunicación visual S. L.

Hussainy, S.; Stykes, K. \& Duncan, G. (2012).A virtual practice environment to develop communication skills in Pharmacy students. American Journal of Pharmaceutical Education, 76 (10) 1-8.

Kopelowicz, A., Liberman, R. P. \& Zarate, R. (2006). Recent advances in social skills training for schizophrenia. Schizophrenia Bulletin, 32, 12-23.

Kroeger, K. A., Schultz, J. R. \& Newsom, C. (2007). A comparison of two groupdelivered social skills programs for young children with autism. Journal of Autism and Developmental Disorders, 37, 808-817.

Lane, C. \& Rollnick, S. (2007). The use of simulated patients and role-play in communication skills training: A review of the literature to August 2005. Patient Education and Counseling, 67, 13-20.

López, S. (2013). Estudio de la efectividad de un entrenamiento en habilidades de comunicación como prevención de la aparición de desgaste profesional en enfermeras de oncología. Proyecto de grado no publicado, Universidad de Coruña, A Coruña, España.

Lozano, A. (2005). El éxito en la enseñanza: aspectos didácticos de las facetas del profesor. México: Trillas.

Meunier, J., Merckaert, I., Libert, Y., Delvaux, N., Etienne, A. M., Liénard, A., et al. (2013). The effect of communication skills training on residents' physiological arousal in a breaking bad news simulated task. Patient education and counseling, 93 (1), 40-47. 
Miczo, N., Segrin, C. \&Allspach, L. E. (2001). Relationship between nonverbal sensitivity, encoding, and relational satisfaction. Communication Reports, 14 (1), 39-48.

Minz, J., Branch, C., March, C. \& Lerman, S. (2012). Key factors mediating the use of a mobile technology tool designed to develop social and life skills in children with Autistic Spectrum Disorders. Computers E Education, 58, 53-62.

Moral, M.V. \& Ovejero, A. (2005). Un programa de intervención psicosocial para la mejora de las habilidades sociales de adolescentes consumidores de alcohol y otras sustancias psicoactivas. Apuntes de Psicología, 23 (1), 3-26.

Mort, J. R. y Hansen, D. J. (2010). First-year Pharmacy Students' Self-Assessment of Communication Skills and the Impact of Video Review. American Journal of Pharmaceutical Education 74 (5), 1-7.

Olaz, F. \& Pérez, E. (2012). Creencias de Autoeficacia: líneas de investigación v desarrollo de escalas. Revista Tesis, 1, 157-170.

Paulley, F. \&Benwari, N. (2014). Repositioning Microteaching for Quality Teacher Production in Nigeria: The Niger Delta University Experience. Journal of Education and Practice, 5 (13), 102-110.

Peñacoba, C.; Ardoy, J.; González-Gutiérrez, J. L.; Moreno-Rodríguez, R. \& Martínez, G. (2003).Efectos de unprograma de habilidades de comunicación en estudiantes de enfermería. Propuesta de uninstrumento de evaluación (ISEC). Clínica y Salud, 14 (2),129-155.

Pereira-Guizzo, C. S., Del Prette, A. \& Del Prette, Z. A. P. (2012). Evaluation of a professional social skills program for unemployed people with physical disability. Psicología: Reflexão e Crítica, 25 (4), 265-274.

Prendes, M. P. \& Solano, I. M. (2008). EDUTEC EN LA RED: Comunidades virtuales para la colaboración de profesionales. EDUTEC. Revista Electrónica de Tecnología Educativa, 25, 1-18.

Rees, C., Sheard, C. \& McPherson, A. (2004).Medical students' views and experiencies of methods of teaching and learning communication skills. Patient Education and Counseling, 54, 119-121.

Roberts, A. \& Ruts, J. (1994). Role and funtion of school psychologist, 1992-93: a comparative study. Psychology in the schools, 31, 113-119.

Sánchez, J. (2013). Búsqueda de Ayuda Académica, Autoeficacia Social Académica y Emociones de Logro en Clase en Estudiantes Universitarios. Revista Argentina de Ciencias del Comportamiento (RACC), 5 (1), 35-41.

Segrin, C. (2000). Social skills deficits associated with depression. Clinical Psychology Review, 20, 379-403.

Segrin, C. \& Flora, J. (2000). Poor social skills are a vulnerability factor in the development of psychosocial problems. Human Communication Research, 26, 489-514.

Tauber, R., Wallace, C. J. \& Leconte, T. (2000).Enlisting indigenous community supporters in skills training programs for persons with severe mental illness. Psychiatric Services, 51, 1428-1432.

Tiuraniemi, J., La“a"ra“, R, Kyro, T. y Lindeman, S. (2011). Medical and psychology students' self-assessed communication skills: A pilot study. Patient Education and Counseling, 83, 152-157. 
Turner, W. A. \& Merriman, L. M. (2005). Clinical skills in treating the foot (2a . Ed). Edinburgh: Elsevier Churchill Livingstone.

Wang, S.Y., Cui, Y. \& Parrila, R. (2011). Examining the effectiveness of peer-mediated and video-modeling social skills interventions for children with autism spectrum disorders: A meta-analysis in single-case research using HLM. Research in Autism Spectrum Disorders, 5, 562-569.

Williams, K. \& Zadro, L. (2001). Ostracism: On being ignored, excluded, and rejected. En M. Leary (Ed.), Interpersonal rejection (pp.21-53). New York: Oxford University Press.

Zade, M. A., Akbari, B. \& Khanzade, A. H. (2014). Effectiveness of training life skills on confronting ability of women who participate in neighborhood house municipality of Tehran, region. Singaporean Journal of Business Economics and Management studies, 2 (8), 43-62. 
